\title{
O relevo na interpretação da variabilidade espacial dos teores de nutrientes em folha de citros
}

Marisol G. A. de Leão ${ }^{1}$, José Marques Júnior ${ }^{1}$, Zigomar M. de Souza ${ }^{2}$, Diego S. Siqueira ${ }^{1 *} \&$ G ener T. Pereira ${ }^{1}$

\section{RESUMO}

A diagnose foliar é um critério para avaliação do estado nutricional das culturas agrícolas, que auxilia na compreensão da fertilidade do solo e na aplicação racional de adubos, levando em conta aspectos econômicos e ambientais. 0 trabalho teve como objetivo utilizar as formas do relevo como regra para auxiliar na interpretação da variabilidade espacial dos teores de nutrientes da folha de citros. Coletas de folhas foram realizadas em interval os regulares de $50 \mathrm{~m}$, totalizando 332 pontos de amostragem. 0 s dados foram analisados pela estatística descritiva, geoestatística e indução da árvore de decisão. Com 0 auxílio do modelo digital de elevação (MDE) e do perfil planialtimétrico, a área foi dividida em diferentes formas do relevo e segmentos de vertente. 0 s maiores valores para os nutrientes da folha de citros foram observados no topo em relação aos segmentos de meia encosta e encosta inferior. 0 s nutrientes da fol ha de citros apresentaram altos valores de correlação $(>0,05)$ com a altitude da área estudada, acima de 0,5 . A técnica de geoestatística e a indução pela árvore de decisão demonstram que o relevo é a variável com maior potencial para interpretar os mapas de variabilidade espacial dos nutrientes da fol ha de citros comparada com outras variáveis de campo como comprimento de rampa e declive.

Palavras-chave: Iaranja, krigagem, mineração de dados, relação solo-paisagem

\section{Landrelief in the interpretation of spatial variability of nutrient content in citrus leaves}

\begin{abstract}
Foliar diagnosis is a method for assessing the nutritional status of agricultural crops, which helps in the understanding of soil fertility and rationalized application of fertilizers taking into account economic and environmental criteria. The study aimed to use the landrelief as criteria to assist in interpreting the spatial variability of nutrient content of the citrus leaf. The leaves were collected at regular intervals of $50 \mathrm{~m}$, totaling 332 sampling points. Data were analyzed by descriptive statistics, geostatistics and induction of decision tree. With the aid of digital elevation model (MDE) and the profile planaltimetric, the area was divided into three different landrelief and sub-strands. The highest values for nutrients from the leaves of citrus were observed at the top (concave area) segments on a half-slope and lower slope. The nutrients from the citrus leaves showed high values of correlation (above 0.5 ) with the altitude of the study area. The technique of geostatistics and the induction of decision tree show that the relief is the variable with the greatest potential to interpret the maps of spatial variability of nutrients from the citrus leaves.
\end{abstract}

Key words: orange, kriging, data mining, soil-landscape relationship

\footnotetext{
${ }^{1}$ FCAV/UNESP, D epartamento de Solos e Adubos, Via de acesso Prof. Paulo Donato Castellane s/n, CEP 14884-900 Jaboticabal, SP. Fone: (16) 3209.2601 E-mail: marisolgal@fcav.unesp.br; marques@fcav.unesp.br; diego_silvasiqueira@yahoo.com.br;genertp@fcav.unesp.br

2FEAGRI/UNICAMP, Campinas, SP. Fone: (19) 3521 1069. E-mail: zigomarms@feagri.unicamp.br
} 


\section{INTRODUÇÃO}

A agricultura moderna carece de uma previsão mais detalhada dos agroecossitemas para realização do manejo sustentável e verificação do efeito desse manejo na qualidade ambiental (Minasny \& Mcbratney, 2007). Martinez et al. (2003) mencionam que para o estabelecimento de um programa apropriado de adubação é necessário identificar e conhecer inúmeras variáveis inerentes à nutrição da planta. Estes autores ressaltam, ainda, que a composição mineral dos tecidos vegetais é influenciada por uma série de fatores pertinentes à própria planta e ao ambiente.

Dentre os fatores ambientais que interferem no rendimento das culturas agrícolas e, portanto, na composição mineral dos tecidos vegetais, destaca-se o relevo (Si \& Farrell, 2004; Kravchenko et al., 2005). Assim, o estudo da relação solo-relevo-planta, é essencial quando se tem, como objetivo, o cultivo sustentável das culturas agrícolas, para evitar a degradação dos atributos químicos, físicos e biológicos do solo.

Entre os critérios para interpretar os resultados da análise foliar se destacam o nível crítico e o Sistema Integrado de Diagnose e Recomendação (DRIS-Diagnosis and Recommendation Integrated System) (Mourão Filho et al., 2002). No Brasil, Mourão Filho \& Azevedo (2004), ressaltaram que o DRIS pode ser uma alternativa para a diagnose nutricional de citros mas deve ser utilizado em conjunto com outras ferramentas que auxiliem o sistema de diagnose.

São encontrados, na literatura, diversos trabalhos sobre os níveis críticos dos nutrientes na folha das culturas agrícolas (Bataglia \& Santos, 1990; Mourão Filho et al., 2002), porém, em muitos casos, esses níveis ao serem estabelecidos não foram consideradas as variações regionais ou locais, sendo oportuno estabelecer padrões de distribuição espacial próprios para cada local, visando a uma avaliação nutricional mais precisa.

$\mathrm{Na}$ literatura se encontram inúmeros trabalhos que caracterizam a variabilidade espacial de atributos químicos e físicos do solo, em menor número a distribuição espacial de pragas e doenças em culturas e a variabilidade de atributos de qualidade de produtos agrícolas. Porém, trabalhos sobre a variabilidade espacial dos teores de nutrientes em folha, são escassos. Souza et al. (1997) ao analisarem a variabilidade dos teores de nutrientes na folha da cultura da laranja, observaram dependência espacial para todos os atributos avaliados, com exceção do $\mathrm{P}$ e $\mathrm{Cu}$.

Ressalta-se também a questão ambiental, o aumento dos custos de produção agrícola, relacionados com a capacidade de aproveitamento das culturas e o excesso do insumo aplicado no solo devido à utilização de superdosagens desses produtos. Sendo o relevo uma variável importante na compreensão da variabilidade espacial da resposta das culturas agrícolas (Martín et al., 2005; Siqueira et al., 2010), o uso de modelos de curvatura pode auxiliar na interpretação da concentração de nutrientes nas folhas da laranja, em escalas mais detalhadas.
Assim, as diferentes formas do relevo identificadas segundo os critérios geomorfológicos, quando associadas a ferramentas de análise como a geoestatística e árvore decisão, podem promover a redução dos custos de produção e minimizar o impacto ambiental. Portanto, o objetivo deste trabalho é utilizar as formas do relevo como critério para auxiliar na interpretação da variabilidade espacial dos teores de nutrientes da folha da planta cítrica.

\section{MATERIAL E MÉTODOS}

A área de estudo está localizada no município de Gavião Peixoto, no Planalto Ocidental Paulista e coordenadas geográficas de $21^{\circ} 75^{\prime}$ 'de latitude sul e $48^{\circ} 46^{\prime}$ de longitude oeste, e altitude variando de 525 a $580 \mathrm{~m}$. O clima da região foi classificado pelo critério de Köppen, como subtropical com inverno seco $(\mathrm{Cwa})$, com precipitação média de $1.400 \mathrm{~mm}$, temperatura média anual de $21^{\circ} \mathrm{C}$, com temperatura no mês mais quente superior a $22{ }^{\circ} \mathrm{C}$ e a do mês mais frio inferior a $18{ }^{\circ} \mathrm{C}$, e umidade relativa do ar de $70 \%$.

A geologia é representada por sedimentos do Grupo Bauru (Formações Adamantina). A porção mais elevada da região é caracterizada pela presença de topos, com encostas convexas de pouca inclinação ( 2 a $5 \%$ ). Essas encostas são interrompidas por ravinas, relativamente profundas, formando vales em "v" aberto, de inclinações escalonadas desde o ombro até os sopés. O solo da área de estudo foi classificado como Latossolo Vermelho distrófico, A moderado, textura média a argilosa seguindo critérios da EMBRAPA (1999).

O pomar de citros em estudo está inserido em uma encosta representativa da região, onde foram identificados e delimitados dois modelos de paisagem. Um com base nas formas do relevo e outro com base nos segmentos de vertente (Campos et al., 2006) (Figura 1). A área de estudo, além da adubação de solo parcelada em três ve-

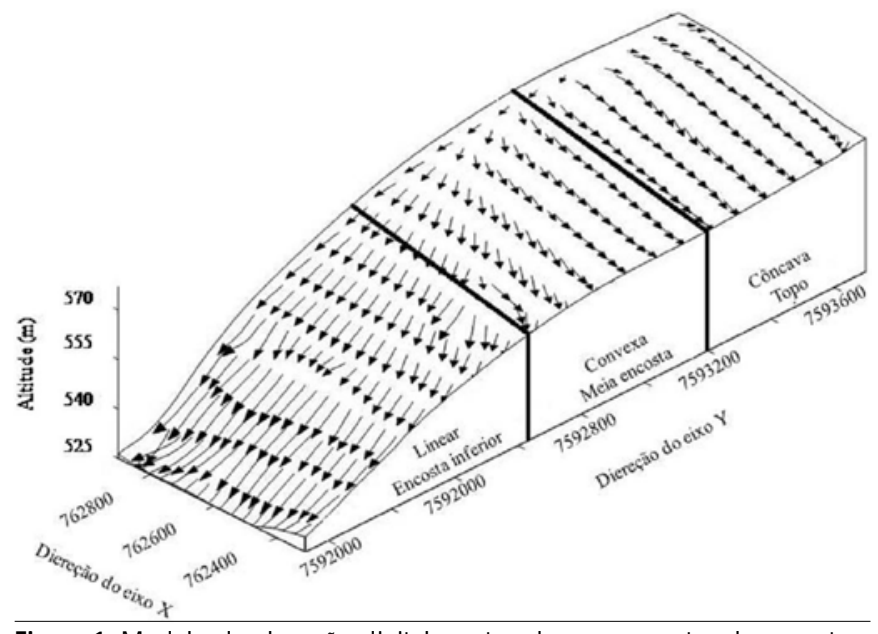

Figura 1. Modelo de elevação digital mostrando os segmentos da encosta e vetores representando o caminhamento superficial (setas)e intensidade (tamanho da seta) dos fluxos de água. No topo e na parte da meia encosta está indicada a área suavemente côncava. 0 s eixos $x$ e y são expressos em coordenadas métricas (Sistema Universal Transverso de Mercator - UTM) 
zes, recebeu quatro pulverizações anuais com adubos foliares. Nas duas vias de adubação a recomendação dos nutrientes foi a mesma, por se tratar de plantas da mesma variedade, idade e com mesma estimativa de produção.

Com a utilização de um aparelho de DGPS foi delimitada uma malha com intervalos regulares de $50 \mathrm{~m}$. Na intersecção dessa malha a planta cítrica foi georreferenciada para coleta das folhas. Coletaram-se amostras de 332 pontos em uma área de 93,6 ha.

Para análise química do tecido vegetal se coletaram, em cada uma das plantas, as terceiras e quartas folhas de ramos vegetativos de cada um dos quadrantes da planta, conforme Mourao Filho et al. (2002). Os macronutrientes $(\mathrm{N}, \mathrm{P}, \mathrm{K}, \mathrm{Ca}$ e $\mathrm{Mg}$ ) e micronutrientes ( $\mathrm{Zn}$ e B) das folhas foram analisados conforme metodologia de Bataglia et al. (1983).

Os resultados dos nutrientes foliares foram avaliados por meio da análise estatística descritiva calculando-se a média, mediana, desvio padrão, coeficiente de variação, coeficiente de assimetria e coeficiente de curtose. A hipótese de normalidade dos dados foi testada pelo teste de Kolmogorov-Smirnov.

Cada nutriente da folha foi submetido ao ajuste de modelos matemáticos na definição dos semivariogramas pelo programa $\mathrm{GS}^{+}$e técnica de interpolação de dados (krigagem), pelo programa Surfer. Para analisar o grau da dependência espacial dos atributos em estudo utilizou-se a classificação de Cambardella et al. (1994), onde são considerados de dependência espacial forte os semivariogramas, que têm efeito pepita $<25 \%$ do patamar, moderada quando está entre 25 e $75 \%$ e fraca $>75 \%$.

$\mathrm{Na}$ análise dos dados por meio da indução da árvore de decisão a variável relevo foi classificada como topo $(\mathrm{T})$, meia encosta (ME) e encosta inferior (EI). Para os nutrientes da folha de citros foram testadas classificações com 3 e 5 níveis, inicialmente pela distribuição de frequência que, na indução da árvore de decisão, não apresentaram resultados satisfatórios. Adotou-se, então, o desvio padrão como critério para classificação e se definiram, como nível médio de produção, os dados no intervalo entre a média mais ou menos um desvio padrão. Os dados inferiores a este intervalo foram definidos como nível baixo e os superiores como nível alto.

Fez-se a indução da árvore de decisão utilizando-se a ferramenta "Tree" ("Tree node") do SAS Enterprise Miner, com algoritmo de redução de entropia. Para evitar que o modelo ficasse muito específico para o conjunto de treinamento, o que comprometeria sua generalização e o desempenho com novos exemplos, adotaram-se duas regras de parada do algoritmo de indução, em que a primeira limitou a profundidade da árvore, o que permite ter, no máximo, seis níveis (nós) e a segunda regra limitou a fragmentação do conjunto de treinamento, requerendo um mínimo de dez exemplos em cada nó para a busca de uma nova divisão e pelo menos cinco exemplos em cada nó folha. A árvore de decisão foi escolhida para ser binária, com dois ramos a partir de cada nó interno. Além das regras de parada, denominadas pré-poda, realizou-se um procedimento de pós-poda, após a indução da árvore completa; avaliaram todas as prováveis subárvores e se escolheu a menor subárvore (menor complexidade).

\section{RESULTADOS E DISCUSSÃO}

Os valores de assimetria e curtose próximos de zero indicam a normalidade de todos os atributos estudados (Tabela 1). A estimação por krigagem apresenta melhores resultados quando a normalidade dos dados é satisfeita. Pelo teste de Kolmogorov-Smirnov observa-se que somente os teores de $\mathrm{N}$ e Ca apresentaram normalidade. Segundo Isaaks \& Srivastava (1989) o coeficiente de assimetria é mais sensível a valores extremos que a média e o desvio padrão, uma vez que um único valor pode influenciar fortemente o coeficiente de assimetria, pois os desvios entre cada valor e a média são elevados a terceira potência.

Tabela 1. Estatística descritiva dos dados dos atributos nitrogênio, fósforo, potássio, cálcio, magnésio, boro e zinco

\begin{tabular}{cccccccc}
\hline Atributos & Média & Mediana & 'DP & Assimetria & Curtose & ${ }^{2}$ CV & ${ }^{3} \mathbf{d}$ \\
$\mathrm{N}$ & 24 & 24 & 0,94 & $-0,03$ & $-0,59$ & 3,93 & $0,05^{\text {ns }}$ \\
$\mathrm{P}$ & 1 & 1 & 0,06 & 0,40 & 0,57 & 5,69 & 0,22 \\
$\mathrm{~K}$ & 12 & 12 & 1,58 & 0,30 & 0,07 & 13,23 & 0,20 \\
$\mathrm{Ca}$ & 32 & 32 & 2,78 & 0,10 & $-0,10$ & 8,58 & $0,08^{\text {ns }}$ \\
$\mathrm{Mg}$ & 2 & 2 & 0,28 & 0,31 & $-0,39$ & 11,22 & 0,15 \\
$\mathrm{~B}$ & 89 & 89 & 9,95 & 0,12 & $-0,20$ & 11,24 & 0,19 \\
Zn & 56 & 55 & 14,20 & 0,17 & $-0,87$ & 25,30 & 0,20 \\
\hline
\end{tabular}

${ }^{1} \mathrm{DP}=$ desvio padrão; ${ }^{2} \mathrm{CV}=$ coeficiente de variação; ${ }^{3} \mathrm{~d}=$ teste de

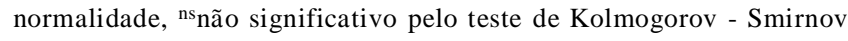

Apesar do teste de normalidade não mostrar distribuições simétricas para os teores de nutrientes da folha da planta cítrica, os valores da média e mediana de todos os atributos estudados são próximos (Tabela 1); isto indica que os dados não apresentam assimetria acentuada. Quando os valores da média e mediana apresentam valores semelhantes, os dados apresentam ou se aproximam da distribuição normal, o que pode ser um indicativo de que as medidas de tendência central não são dominadas por valores atípicos na distribuição (Cambardella et al., 1994), mostrando que os dados estão adequados para uso da geoestatística.

Os valores do coeficiente de variação (CV) dos teores de nutrientes da folha de citros indicam altos valores para Zn, segundo a classificação de Warrick \& Nielsen (1980), valores médios para o $\mathrm{K}$ e baixo coeficiente de variação para os demais atributos estudados (Tabela 1). Souza et al. (1997) analisaram a variabilidade dos teores de nutrientes da folha de citros e obtiveram baixo $\mathrm{CV}$ para o $\mathrm{N} \mathrm{e}$ mediano para $\mathrm{Mg}, \mathrm{Mn}, \mathrm{Ca}, \mathrm{P}$ e $\mathrm{Zn}$.

Observa-se alta correlação, acima de 0,5 , entre os nutrientes da folha da planta cítrica e a topografia do terreno, com exceção do $\mathrm{Mg}$ (Tabela 2). Os atributos $\mathrm{N}$ e $\mathrm{P}$ apresentaram correlação com locais de maiores altitudes 
Tabela 2. Matriz de correlação linear entre altitude da área de estudo e os nutrientes da folha de citros

\begin{tabular}{|c|c|c|c|c|c|c|c|c|}
\hline & Altitude & $\mathbf{N}$ & $P$ & K & $\mathrm{Ca}$ & $\mathrm{Mg}$ & B & $\mathrm{Zn}$ \\
\hline Altitude & 1 & - & - & - & - & - & - & - \\
\hline $\mathrm{N}$ & $0,80 *$ & 1 & - & - & - & - & - & - \\
\hline$P$ & $0,71 *$ & $0,67^{*}$ & 1 & - & - & - & - & - \\
\hline K & $0,64 *$ & $0,58^{*}$ & $0,43^{\text {ns }}$ & 1 & - & - & - & - \\
\hline $\mathrm{Ca}$ & $0,62 *$ & 0,22 ns & 0,28 ns & $-0,26$ ns & 1 & - & - & - \\
\hline $\mathrm{Mg}$ & 0,44 ns & 0,13 ns & 0,24 ns & $-0,24$ ns & $0,53^{*}$ & 1 & - & - \\
\hline B & $0,51^{*}$ & 0,36 ns & 0,33 ns & 0,08 ns & 0,18 ns & 0,12 ns & 1 & - \\
\hline Zn & $0,53^{*}$ & 0,20 ns & $0,15^{\text {ns }}$ & 0,22 ns & 0,02 ns & $0,04^{\text {ns }}$ & $0,18^{\mathrm{ns}}$ & 1 \\
\hline
\end{tabular}

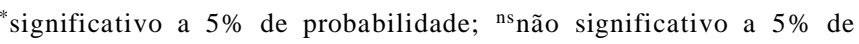
probabilidade

na área (acima de 0,70). A declividade do terreno revelou correlação positiva com os nutrientes da folha. Kravchenko \& Bullock (2002) também encontraram correlações significativas entre o relevo e os atributos da planta, em dois anos de estudo. Segundo Kravchenko \& Robertson (2005) em uma escala local a topografia do terreno e o relevo têm sido considerados a mais importante variável na distribuição espacial de culturas porque, comumente, ela corresponde às mudanças nos atributos dos solos.

Todas as variáveis analisadas apresentaram dependência espacial, expressa pelos modelos de semivariogramas

Tabela 3. Modelos e parâmetros estimados dos semivariogramas experimentais para os atributos nitrogênio, fósforo, potássio, cálcio, magnésio, boro e zinco

\begin{tabular}{ccccccc}
\hline Atributos & Modelo & Efeito Pepita & Patamar & Alcance & ${ }^{1}$ GDE & ${ }^{2} \mathbf{R}^{2}(\%)$ \\
N & Exponencial & 0,33 & 0,74 & 462 & 45 & 96 \\
P & Exponencial & 0,001 & 0,003 & 222 & 30 & 96 \\
K & Esférico & 0,47 & 2,22 & 431 & 21 & 92 \\
Ca & Exponencial & 1,71 & 7,05 & 303 & 24 & 93 \\
Mg & Esférico & 0,03 & 0,08 & 291 & 33 & 96 \\
B & Esférico & 36,50 & 91,4 & 373 & 40 & 98 \\
Zn & Esférico & 29,10 & 211,00 & 385 & 14 & 97 \\
\hline
\end{tabular}

${ }^{1} \mathrm{GDE}=\mathrm{C}_{0} /\left(\mathrm{C}_{0}+\mathrm{C}_{1}\right)$, grau de dependência espacial $(\%) ;{ }^{2} \mathrm{R}^{2}=$ coeficiente de determinação $(\%)$

(Tabela 3). As variáveis se ajustaram ao modelo esférico e exponencial (Isaaks \& Srivastava, 1989). A análise da relação $\mathrm{C}_{0} /\left(\mathrm{C}_{0}+\mathrm{C}_{1}\right)$ mostrou que todas as variáveis apresentaram grau de dependência espacial moderada, com exceção da variável Zn, que apresentou grau de dependência espacial forte. Houve dois grupos de alcance para os atributos em estudo, aqueles com variação entre 222 $\mathrm{m}$ a $303 \mathrm{~m}$ ( $\mathrm{P}, \mathrm{Mg}$ e $\mathrm{Ca})$ e o grupo com alcance variando entre $373 \mathrm{~m}$ a $1008 \mathrm{~m}(\mathrm{~B}, \mathrm{Zn}, \mathrm{K}$ e N ).

$\mathrm{O} \mathrm{N}$ apresenta pequena variação nos teores foliares de 22 a $26 \mathrm{~g} \mathrm{~kg}^{-1}$ (Figura 2A). Segundo Malavolta et al. (1997) o teor de $\mathrm{N}$ foliar adequado para a cultura de citros é de 22 a $27 \mathrm{~g} \mathrm{~kg}^{-1}$; assim, toda a área se encontra com teores de $\mathrm{N}$ suficientes. No mapa de distribuição espacial do $\mathrm{N}$ nota-se uma faixa com altos valores que ocupa a parte mais elevada do segmento I (topo - área côncava). Os teores de $\mathrm{N}$ diminuem no sentido do topo para a encosta inferior, concordando com os valores obtidos pela correlação positiva entre $\mathrm{N}$ e altitude (Tabela 2).

No mapa de distribuição espacial do atributo $\mathrm{P}$ observa-se que os teores do nutriente variaram de 0,93 a 1,13 $\mathrm{g} \mathrm{kg}^{-1}$ (Figura 2B), teores considerados baixos por Malavolta et al. (1997). Nesse mapa, como é normalmente encontrado em resultados referentes a este elemento no solo, nota-se uma variabilidade maior dos dados (Salviano et al., 1998). Em relação ao relevo, a área côncava do topo mostrou os maiores valores para o $\mathrm{P}$, estando de acordo com a correlação dos dados, que foi de 0,71 (Tabela 2).

Os teores do atributo $\mathrm{K}$ na área de estudo variaram entre 10 e $15 \mathrm{~g} \mathrm{~kg}^{-1}$ (Figura 2C), segundo Malavolta et al. (1997) teores de 10 a $17 \mathrm{~g} \mathrm{~kg}^{-1}$ são considerados ideais para a cultura de citros; assim, a maior parte da área estudada se encontra nesta faixa. A área côncava do topo apresenta os maiores teores para o $\mathrm{K}$, comportamento observado na correlação deste atributo com a topografia, 0,64 (Tabela 2).

Os teores de Ca no mapa de distribuição espacial variaram entre 27 a $36 \mathrm{~g} \mathrm{~kg}^{-1}$ (Figura 2D), considerados nível baixo $\left(<29 \mathrm{~g} \mathrm{~kg}^{-1}\right)$. O nível ótimo se encontra entre $30 \mathrm{a}$ $55 \mathrm{~g} \mathrm{~kg}^{-1}$ segundo Malavolta et al. (1997), estando a grande maioria da área dentro dos níveis ideais. $\mathrm{O} \mathrm{Ca}$, juntamente com o $\mathrm{N}$ e o K, são os nutrientes constituintes mais encontrados na biomassa de citros de acordo com Mattos Júnior et al. (2003). A área côncava do topo apresentou os maiores valores para o $\mathrm{Ca}$ e os menores se acham no segmento da meia encosta e encosta inferior, de acordo, portanto, com a correlação simples entre $\mathrm{Ca}$ e a altitude da área, que foi superior a 0,50.

Os teores de $\mathrm{Mg}$ na folha variaram de 2,0 a $3,1 \mathrm{mg} \mathrm{kg}^{-1}$ (Figura 2E), enquanto a faixa ideal é de 2,6 a $6,0 \mathrm{mg} \mathrm{kg}^{-1}$ (Malavolta et al., 1997); desta forma, pelo mapa de $\mathrm{Mg}$ nota-se que a maior parte da área de estudo se situa com níveis baixos deste nutriente. Observando os mapas de isolinhas do $\mathrm{Mg}$ e do Ca é possível visualizar que, apesar do contorno das isolinhas nos dois mapas serem diferentes, eles guardam semelhanças em relação ao comportamento na encosta. Para o $\mathrm{Mg}$ não se observa uma diferenciação pelo mapa de distribuição espacial dos maiores e menores valores ao longo da vertente, porém a correlação deste atributo com o relevo foi de 0,44 .

No mapa de distribuição espacial do boro, os teores desse nutriente variaram de 72 a $104 \mathrm{mg} \mathrm{kg}^{-1}$ (Figura 2F), a faixa considerada ideal é de 30 a $100 \mathrm{mg} \mathrm{kg}^{-1}$ (Malavolta et al., 1997). Os altos valores para o B se encontram no topo, área suavemente côncava; no restante da área, incluindo-se parte do topo, os valores são mais homogêneos, enquanto na meia encosta e encosta inferior predominam os valores intermediários e baixos.

$\mathrm{O} \mathrm{Zn}$ e o B são, juntos, os micronutrientes com os quais os citricultores mais se preocupam em acompanhar via análise foliar. Os teores de $\mathrm{Zn}$ considerados ótimos estão entre 25 a $100 \mathrm{mg} \mathrm{kg}^{-1}$ (Malavolta et al., 1997), e os valores apresentados na área variam entre 30 e $80 \mathrm{mg} \mathrm{kg}^{-1}$ (Figura 2G). Em relação ao relevo no mapa de distribui- 
A.

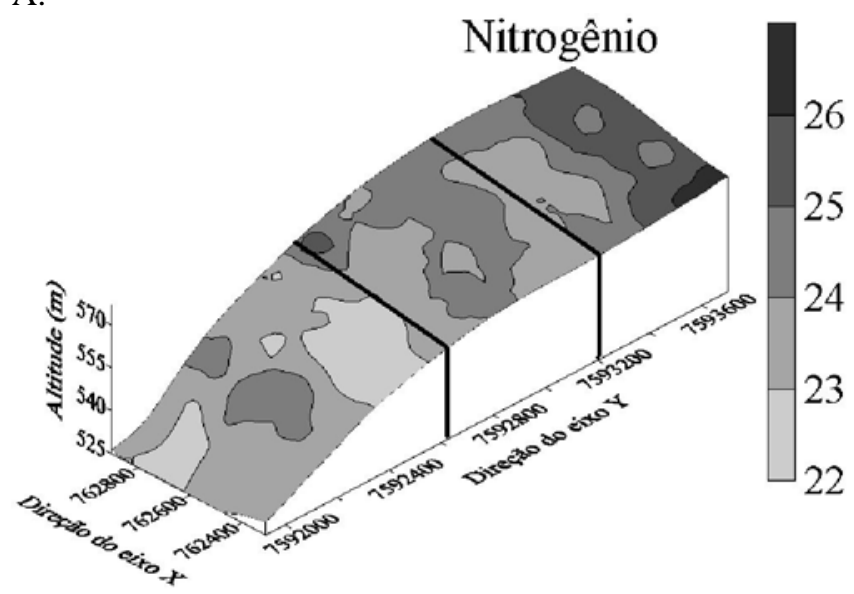

C.

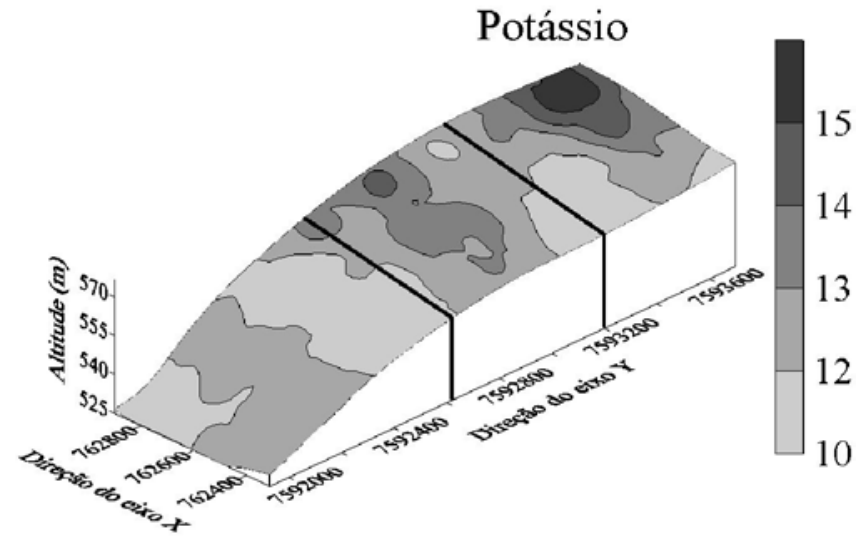

E.

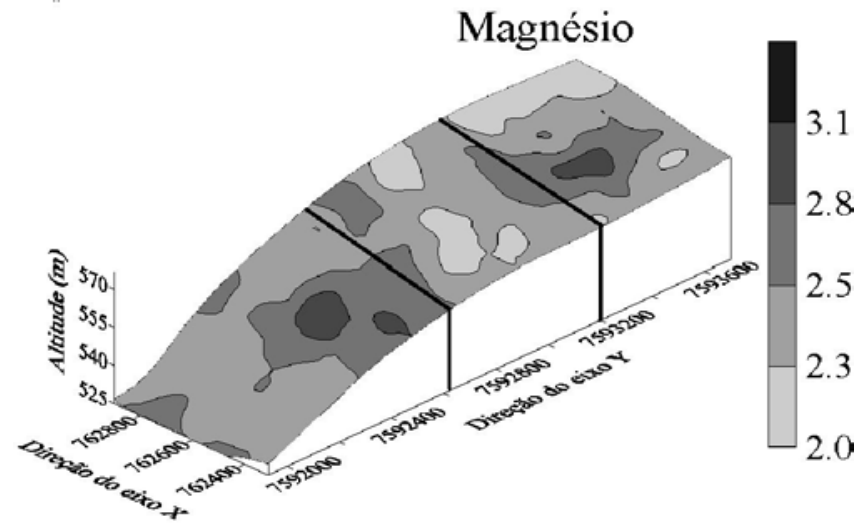

B.

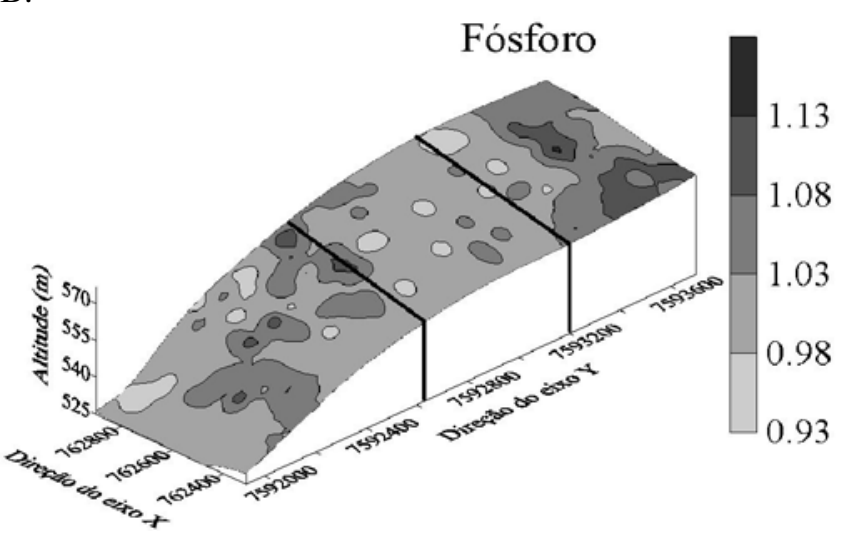

D.

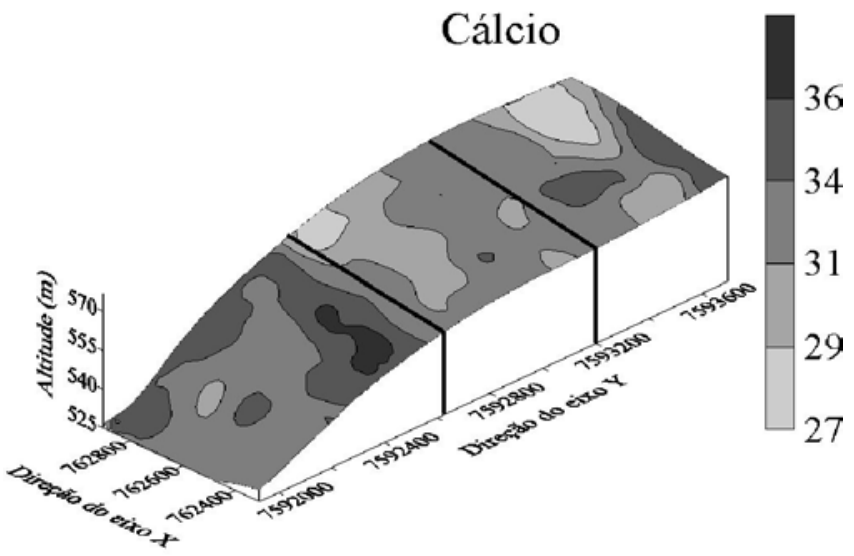

F.

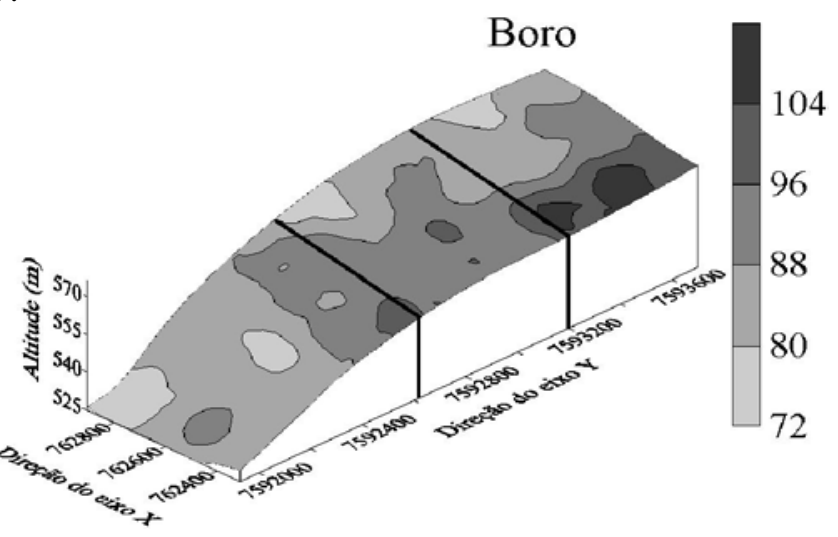

G.

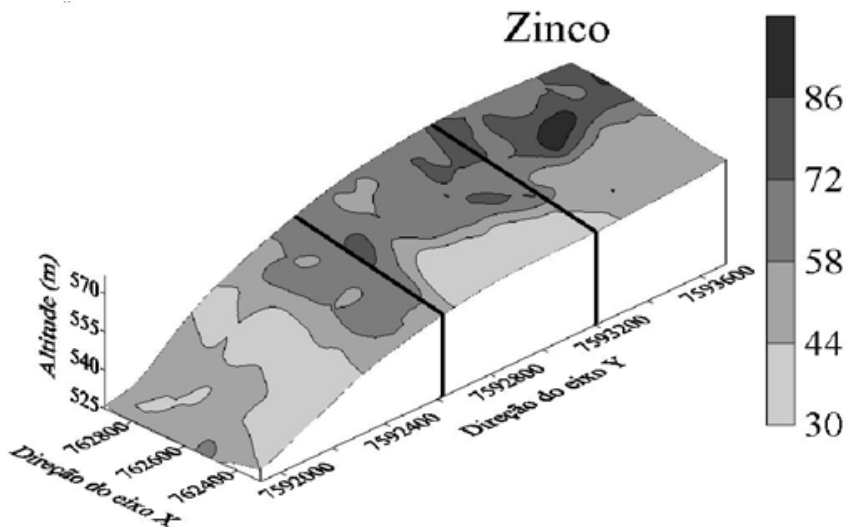

Figura 2. Distribuição espacial dos atributos nitrogênio $(\mathrm{N})$, fósforo (P), potássio $(\mathrm{K})$, cálcio $(\mathrm{Ca})$, magnésio $(\mathrm{Mg})$, boro $(\mathrm{B})$ e zinco $(\mathrm{Zn})$, em mg kg-1 


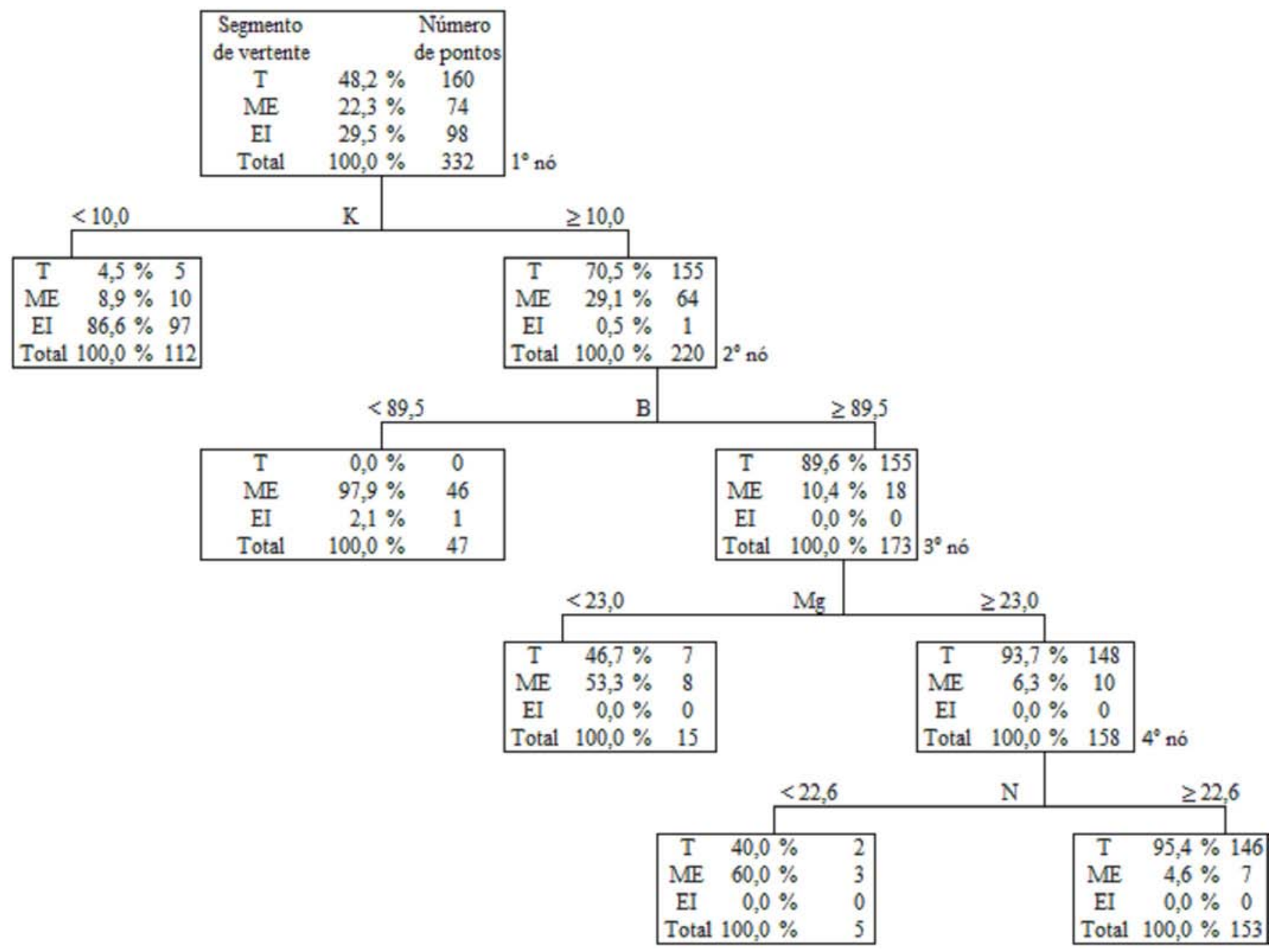

Figura 3. Á rvore de dec isão que auxilia na compreensão da variabilidade dos nutrientes da fol ha de citros, de acordo com os segmentos de vertente. As informações em cada nó representam, de cima para baixo, o número identificador do nó, os segmentos de vertente predominante ea distribuição percentual de cada classe no nó. O s nós são coloridos em tons de cinza com base na proporção de exemplos da classe. T-topo; M E-meia encosta; El- encosta inferior

ção espacial do Zn observa-se concentração dos teores mais elevados no topo da área côncava; depois, há uma área com valores menores localizados na meia encosta e, na encosta inferior, predominam os valores intermediários e baixos.

A árvore de decisão gerada ajuda a compreender a relação entre as variáveis da planta com os segmentos de encosta identificados na área (Figura 3). A primeira variável usada na decisão para os diferentes segmentos de encosta (topo - área côncava, meia encosta - convexa e encosta inferior - linear) foi o K, definindo a distribuição dos maiores e menores para o $\mathrm{K}$ em relação os segmentos de encosta, os menores valores para $\mathrm{K}$ se encontram na encosta inferior e os maiores na área côncava com uma pequena quantidade na meia encosta. A partir do $2^{\circ}$ nó na árvore decisão, com $\mathrm{K}$ da folha $\geq 10,0 \mathrm{~g} \mathrm{~kg}^{-1}$, observouse que os maiores valores para o $\mathrm{B}, \mathrm{Mg}$ e $\mathrm{N}$ ocorreram no topo do terreno (área côncava).

A árvore de decisão induzida demonstrou a influência do relevo nos nutrientes da folha de citros, para a área estudada (Figura 3). Dentre um conjunto de atributos da folha de citros, associados com relevo, a árvore induzida estabelece limites do relevo com alta correlação com os níveis dos nutrientes da folha citros. Para o K, os valores menores que $10,0 \mathrm{~g} \mathrm{~kg}^{-1}$ foram observados na encosta inferior, com $86,6 \%$ de acerto; já para os valores maiores que $10,0 \mathrm{~g} \mathrm{~kg}^{-1}$, se encontram em grande parte na área côncava - topo ( $71 \%$ de acerto) e o restante na meia encosta (29\% de acerto). Quando os valores de B foram maiores ou iguais a $89,5 \mathrm{mg} \mathrm{kg}^{-1} 90 \%$ dos pontos amostrais se localizavam na área côncava (topo) e $10 \%$ na meia encosta; para o $\mathrm{Mg}$ e $\mathrm{N}$, a árvore de decisão demonstra ocorrência dos maiores valores na área côncava, com $94 \%$ e $96 \%$ de acerto, respectivamente.

Observa-se, por meio da correlação simples da técnica de geoestatística e pela indução da árvore de decisão, que o relevo da área em estudo apresentou boa correlação com os nutrientes da folha da cultura do citros, entre $0,44 \mathrm{e}$ 0,88 (Tabela 2). Estudos demonstram que o relevo tem sido uma variável que define a distribuição de atributos do 
solo e da planta ao longo da paisagem e tem apresentado altas correlações com a produtividade de culturas e atributos do solo (Iqbal et al., 2005; Siqueira et al., 2010).

Verifica-se, dentro da área suavemente côncava (topo), um padrão de comportamento seguindo as rotas preferenciais de água com a ocorrência dos maiores valores dos atributos avaliados neste segmento de vertente. Segundo Resende et al. (1997) a forma de relevo côncava favorece a concentração de água no sistema e apresenta menor perda de solo em relação às formas convexas (meia encosta) e lineares (encosta inferior), com maiores teores de nutrientes no solo e na planta. Estudos têm demonstrado que as áreas convexas e lineares apresentam variabilidade maior para atributos do solo e da planta em relação às áreas côncavas, concordando com os resultados obtidos neste trabalho nas diferentes formas de se avaliar as variabilidades dos atributos estudados (Montanari et al., 2005; Camargo et al., 2008).

\section{CONCLUSÕES}

1. Os maiores valores para os nutrientes da folha de citros foram observados no topo (área côncava) em relação aos segmentos de meia encosta e encosta inferior.

2. Os nutrientes da folha de citros apresentaram altos valores $(>0,5)$ de correlação com a altitude da área estudada.

3. A técnica de geoestatística e a indução pela árvore de decisão demonstram que as formas relevo podem auxiliar na interpretação dos mapas de variabilidade espacial dos nutrientes da folha da planta cítrica e identificação de zonas de manejo específico.

\section{LITERATURA CITADA}

Bataglia, O. C.; Furlani, A. M. C.; Teixeira, J. P. F.; Furlani, P. R.; Gallo, J. R. Métodos de análise química das plantas. Boletim do Instituto Agronômico de Campinas, v.78, 1983, p.48.

Bataglia, O. C.; Santos, W. R. dos. Efeito do procedimento de cálculo e da população de referência nos índices do sistema integrado de diagnose e recomendação (DRIS). Revista Brasileira de Ciência do Solo, v.14, p.339-344, 1990.

Camargo, L. A.; Marques Júnior, J.; Pereira, G. T.; Horvat, R. A. Variabilidade espacial de atributos mineralógicos de um latossolo sob diferentes formas do relevo. II - Correlação espacial entre mineralogia e agregados. Revista Brasileira de Ciência do Solo, v. 32, p. 2279-2288, 2008.

Cambardella, C. A.; Moorman, T. B.; Novak, J. M.; Parkin, T. B.; Karlen, D. L.; Turco, R. F.; Konopka, A. E. Field scale variability of soil properties in Central Iowa soils. Soil Science Society of America Journal, v.58, p.1501-1511, 1994.

Campos, M. C. C.; Cardozo, N. P.; Marques Júnior, J. . Modelos de paisagem e sua utilização em levantamentos pedologicos. Revista de Biologia e Ciências da Terra, v.6, p.104-114, 2006.

EMBRAPA - Empresa Brasileira de Pesquisa Agropecuária. Centro Nacional de Pesquisa de Solos. Sistema Brasileiro de Classificação de Solos. Rio de Janeiro: EMBRAPA CNPS, 1999. p.412.
Iqbal, J.; Read, J. J.; Thomasson, A. J.; Jenkins, J. N. Relationships between Soil-Landscape and Dryland Cotton Lint Yield. Soil Science Society of America Journal, v.69, p.872-882, 2005.

Isaaks, E. H.; Srivastava, R. M. An introduction to applied geoestatistics. New York: Oxford University Press, 1989. $561 \mathrm{p}$.

Kravchenko, A. N.; Bullock, D. G. Correlation of corn and soybean yield with topography and soil properties. Agronomy Journal, v.75, p.75-83, 2002.

Kravchenko, A. N.; Robertson, G. P.; Thelen, K. D.; Harwood, R. R. Management, Topographical, and Weather Effects on Spatial Variability of Crop Grain Yields. Agronomy Journal, v.97, p.514-523, 2005.

Malavolta, E.; Vitti, G. C.; Oliveira, S. A. Avaliação do estado nutricional das plantas: princípios e aplicações. Piracicaba: Associação Brasileira para a Pesquisa da Potassa e do Fosfato, 1997. 319p.

Martín, N. F.; Bollero, G. A.; Bullock, D. G. Associations between field characteristics and soybean plant performance using canonical correlation analysis. Plant and Soil, v.273, p.39-55, 2005.

Martinez, H. E. P.; Menezes, J. F. S.; Souza, R. B.; Venegas, V. H. A.; Guimarães, P. T. G. Faixas críticas de concentrações de nutrientes e avaliação do estado nutricional de cafeeiros em quatro regiões de Minas Gerais. Pesquisa Agropecuária Brasileira, v.38, p.703-713, 2003.

Mattos Júnior. D.; Quaggio, J. A.; Cantarella, H.; Alva, A. K. Conteúdo de nutrientes em componentes da biomassa de laranjeira hamlin. Scientia Agrícola, v.60, p.155-160, 2003.

Minasny, B.; Mcbratney, B. Incorporating taxonomic distance into spatial prediction and digital mapping of soil classes. Geoderma, v.142, p.285-293, 2007.

Montanari, R.; Marques Júnior, J.; Pereira, G. T.; Souza, Z. M. Forma da paisagem como critério para otimização amostral de latossolos sob cultivo de cana-de-açúcar. Pesquisa Agropecuária Brasileira, v.40, p.69-77, 2005.

Mourão Filho, F. A. A.; Azevedo, J. C. DRIS: concepts and applications on nutritional diagnosis in fruit crops. Scientia Agricola, v.61, p.550-560, 2004.

Mourao Filho, F. A. A.; Azevedo, J. C.; Nick, J. A. Funções e ordem da razão dos nutrientes no estabelecimento de normas DRIS em laranjeira 'Valência'. Pesquisa Agropecuária Brasileira, v.37, p.185-192, 2002.

Resende, M.; Curi, N.; Rezende, S. B.; Corrêa, G. F. Pedologia: Base para distinção de ambientes. 2.ed. Viçosa: NEPUT, 1997. $367 \mathrm{p}$.

Salviano, A. A. C.; Vieira, S. R.; Sparovek, G. Variabilidade espacial de atributos de solo e de Crotalaria juncea (L.) em área severamente erodida. Revista Brasileira de Ciência do Solo, v.22, p.115-122, 1998.

Si, B. C.; Farrell, R. E. Scale-dependent relationship between wheat yield and topographic indices: A wavelet approach. Soil Science Society of America Journal, v.68, p.577-587, 2004.

Siqueira, D. S.; Marques Júnior, J.; Pereira, G. T. The use of landforms to predict the variability of soil and orange attributes. Geoderma, v.155, p.55-66, 2010. 
Souza, L. S.; Cogo, N. P.; Vieira, S. R. Variabilidade dos teores de nutrientes na folha, em um pomar cítrico. Revista Brasileira de Ciência do Solo, v.21, p.373-377, 1997.
Warrick, A. W.; Nielsen, D. R. Spatial variability of soil physical properties in the field. In: Hillel, D. (ed.). Applications of soil physics. New York: Academic Press, 1980. p.319-344. 